\title{
SNP rs2240688 in CD133 gene on susceptibility and clinicopathological features of hepatocellular carcinoma
}

\author{
Xiaolan Pan ${ }^{1 \#}$, Lingsha Huang ${ }^{1 \#}$, Dan $\mathrm{Mo}^{2}$, Yihua Liang ${ }^{1}$, Zhaodong Huang ${ }^{1}$, Bo Zhu ${ }^{1}$, Min Fang ${ }^{1}$ \\ ${ }^{1}$ Department of Clinical Laboratory, Guangxi Medical University Affiliated Tumor Hospital, Nanning, China; ${ }^{2}$ Department of Surgery, Maternal and \\ Child Health Hospital of the Guangxi Zhuang Autonomous Region, Nanning, China \\ Contributions: (I) Conception and design: M Fang; (II) Administrative support: B Zhu; (III) Provision of study materials or patients: L Huang; (IV) \\ Collection and assembly of data: X Pan; (V) Data analysis and interpretation: X Pan; (VI) Manuscript writing: All authors; (VII) Final approval of \\ manuscript: All authors. \\ \#These authors contributed equally to this work. \\ Correspondence to: Min Fang; Bo Zhu. Department of Clinical Laboratory, Guangxi Medical University Affiliated Tumor Hospital, Nanning, China. \\ Email: sunflowersfun@126.com; zhubogxnn@126.com.
}

Background: CD133 is one of the important cancer stem cells (CSCs) markers of hepatocellular carcinoma (HCC). The aim of this study was to explore the relationship between CD133 single-nucleotide polymorphisms (SNPs) and risk factors associated with HCC susceptibility and clinicopathological features in HCC cases and healthy controls from the Guangxi region of southern China.

Methods: A case control study was conducted, including 565 HCC patients and 561 control subjects. The genotyping of rs2240688 was performed using the SNaPshot method. Unconditional logistic regression was used to correct for gender, age, and other confounding factors. Odds ratio (OR) and its $95 \%$ confidence interval (CI) were calculated to analyze the relationship between allele and genotype frequency and the risk of HCC.

Results: The distribution frequencies of CD133 alleles and genotypes in the HCC case group and the control group were statistically significant $(\mathrm{P}<0.05)$. The CA heterozygous $(\mathrm{P}=0.003, \mathrm{OR}=1.463,95 \%$ CI: $1.134-1.887)$ and $C C$ homozygous genotypes $(\mathrm{P}=0.036, \mathrm{OR}=1.910,95 \% \mathrm{CI}: 1.044-3.493)$, as well as $\mathrm{C}$ carrier status $(\mathrm{P}=0.004, \mathrm{OR}=1.465,95 \% \mathrm{CI}$ : $1.136-1.889)$ and $\mathrm{C}$ alleles $(\mathrm{P}=0.004, \mathrm{OR}=1.465,95 \%$ CI: 1.136-1.889), were associated with an increased risk of HCC. Additionally, in the subgroup analysis of CD133 rs2240688 polymorphism and clinical characteristics, the results showed that the genotype distribution of CD133 rs2240688 was significantly different in genotype distribution of metastasis and alanine aminotransferase (ALT).

Conclusions: the expression of miRNA binding site rs2240688 of tumor stem cell marker gene CD133 in HCC may be a promising marker for the prediction of HCC, but larger studies are still needed.

Keywords: Hepatocellular carcinoma (HCC); single-nucleotide polymorphisms (SNPs); cancer stem cells (CSCs); CD133; rs2240688

Submitted Dec 04, 2019. Accepted for publication Aug 21, 2020.

doi: $10.21037 /$ tcr-19-2690

View this article at: http://dx.doi.org/10.21037/tcr-19-2690

\section{Introduction}

Primary liver cancer has the fifth highest incidence rate of malignant tumors worldwide, and is the second leading cause of male mortality (1). The estimated annual global incidence of primary liver cancer is 841,000 , and the number of deaths is estimated to be 782,000 (1). In China, liver cancer has the second highest mortality rate of malignant tumors, and new liver cancer cases account for more than $50 \%$ of the world's total, increasing year by year (2). There are several risk factors for liver cancer, including viral infection, heredity, aflatoxin contamination, 
carcinogen exposure, non-fatty alcoholic hepatitis, and various single-nucleotide polymorphisms (SNPs) (1-3). Despite the advancements in technology and improvements in treatments including surgery, radiotherapy, chemotherapy, and the use of other biological agents, the prognosis of hepatocellular carcinoma (HCC) is poor due to recurrence and metastasis, with a 5 -year disease-free survival rate of $16 \%$ to $27.1 \%$ (4).

Several studies have demonstrated that cancer stem cell (CSCs) subgroups, whose functions are responsible for tumor persistence and recurrence, metastasis, drug resistance, and radiation tolerance, may drive tumorigenesis $(5,6)$. CD133 (prominin-1) is a 5-transmembrane glycoprotein expressed on a subset of hematopoietic stem cells derived from fetal liver and bone marrow. CD133 is considered to be a CSC marker for a variety of cancer types, including HCC $(7,8)$, colon cancer (9), gastric cancer (10), and ovarian cancer (11). It is associated with higher colony formation efficiency, a greater proliferation rate, and higher tumor incidence (12). Studies have found that HCC patients with elevated CD133 levels have a lower overall survival rate and higher recurrence rates than patients with lower CD133 expression levels. Although there are several studies on certain susceptibility genes for HCC (13-15), studies on CD133 SNPs in the context of HCC susceptibility and clinical features are still lacking. Furthermore, polymorphisms in the CD133 gene have been associated with a variety of human diseases (16-18). Given the limited number of studies examining CD133 polymorphisms in HCC, we investigated the association between SNP rs2240688 and the demographics, clinical features, and prognosis of HCC in a Chinese population.

\section{Methods}

\section{Study population}

Subjects for this case control study were recruited from the Affiliated Tumor Hospital of Guangxi Medical University between September 2016 to December 2018. All participants received a relevant questionnaire in order to collect information on the history of environmental exposure after signing written informed consent. The demographic data collected included medical record number, gender, age, drinking status, smoking status, histological tumor type, tumor-node-metastasis stage, related biochemical indicators, and other information. In order to avoid selection bias, inclusion criteria, such as age and gender, were matched between the control group and the case group. The control group was recruited continuously from December 2018 to February 2019 from the physical examination center of the First Affiliated Hospital of Guangxi Medical University. Meanwhile, the control group comprised healthy subjects who had good daily life function, and no heart disease, cerebrovascular disease, infectious disease, autoimmune disease, abnormal physical examination indexes, or a personal or family history of cancer. Written informed consent was provided by all subjects in the study. The study was conducted in accordance with the Declaration of Helsinki (as revised in 2013). This study was approved by the ethics committee of the Affiliated Tumor Hospital of Guangxi Medical University (approval ID: LW2020007).

\section{DNA extraction and genotyping assays}

Blood samples (2 $\mathrm{mL})$ from each subject were collected and placed in an EDTA-K2 anticoagulant tube, thoroughly mixed, and stored at $-20^{\circ} \mathrm{C}$. Genomic DNA was isolated using a commercial kit (Adelaide, Beijing, China) according to the manufacturer's instructions. The genotyping of CD133 rs2240688 was performed using the SNaPshot method (19), and, in order to ensure the accuracy of genotype evaluation, a negative control was used for each test. The forward primer sequence for CD133 rs2240688 polymorphism was 5'-CTCATGTTAGCTGCACTCCAAT-3', and the reverse primer sequence for CD133 rs2240688 polymorphism was 5'-ACCATTGACTTCTTGGTGCTG-3' (328 bp).

\section{Statistical analysis}

In order to confirm the representativeness of the population of study samples, a Chi-square test was used to determine whether the samples conformed to the Hardy-Weinberg Equilibrium (HWE) law. When the $\mathrm{P}$ value $>0.05$, the samples were considered to be representative of the population. Two independent sample Chi-square tests were used to test the difference between the two groups. Differences in genotype and allele frequency between the HCC and control groups were assessed using a Chisquare test with Bonferroni correction. Chi-square testing and logistic regression analyses were used to compare the distribution data of alleles and genotypes, and the relative risk was expressed as an odds ratio (OR) and its $95 \%$ confidence interval (CI). The logistic regression method 
was applied to correct for the effects of confounding factors such as gender and age. All statistical tests were performed using SPSS 24.0 (SPSS Inc., Chicago, IL, USA), and were two-sided, with a $\mathrm{P}$ value $<0.05$ considered to be statistically significant.

\section{Results}

\section{Baseline characteristics of the study population}

Initially, 593 subjects were enrolled in the HCC group, and 561 subjects were enrolled in the control group. There were 21 cases without pathological reports and 7 cases with liver metastases records, which were excluded from the HCC group. Ultimately, 565 HCC patients and 561 controls were included in this study, and their clinical parameters are presented in Table 1. We analyzed the demographic characteristics of the subjects and found that the mean age, gender, and body mass index (BMI) classification of the two groups of patients were matched. The average age of patients with HCC was 53.62 years, ranging from $10-89$ years. Similarly, the average age of the control group was 52.15 years, ranging from 22-78 years. Interestingly, the majority of patients were male $(86.19 \%)$. After statistical analysis, the age and gender of HCC patients were not significantly different from those of the control group $(\mathrm{P}>0.05)$.

\section{CD133 rs2240688 polymorphism and HCC risk}

The genotype frequency of CD133 rs2240688 was consistent with the Hardy-Weinberg equilibrium law, indicating that the samples selected in this study were representative of the population of interest. The most frequently distributed allele in the controls and recruited HCC patients was AA heterozygous. The genotype frequencies of the CD133 rs2240688 locus in the HCC group were 333 (58.9\%) for AA, 202 (35.7\%) for CA, and $30(5.3 \%)$ for CC. Similarly, the genotype frequency distribution of this locus in the control group was 384 (68.4\%) for AA, 159 (28.3\%) for CA, and 18 (3.2\%) for CC. The frequency distribution of the AA, CA, and CC genotypes between the two groups was statistically significant $(\mathrm{P}<0.001)$. In the overall analysis, multiple comparisons using a Chi-square test with Bonferroni correction found that the distribution of the AA genotype was different from that of the CA and CC genotype, and the distribution of the A allele also differed from that of the $\mathrm{C}$ genotype $(\mathrm{P}=0.0167)$. We then used the AA genotype and A allele as a reference to analyze the risk of HCC. For comparing genotypes and alleles of HCC susceptibility, the logistic regression model of the two categorical variables was used to correct for the influence of confounding factors such as gender and age, and the OR value and $95 \%$ CI of rs2240688 on the risk of liver cancer were calculated. Individuals carrying the rs2240688 CA + CC genotype had a 1.508 -fold higher risk of developing HCC than individuals carrying the AA genotype $(\mathrm{P}<0.001, \mathrm{OR}=1.910$, 95\% CI: 1.181-1.926). Similarly, individuals carrying the homozygous CC genotype were 1.910 times more likely to develop HCC than individuals carrying the AA genotype $(\mathrm{P}=0.036, \mathrm{OR}=1.910,95 \% \mathrm{CI}: 1.044-3.493)$. Individuals carrying the heterozygous CA genotype were 1.463 times more likely to develop HCC than individuals carrying the AA genotype ( $\mathrm{P}=0.003$, OR $=1.463,95 \%$ CI: $1.134-1.887$ ). In addition, individuals carrying the $\mathrm{C}$ allele were at 1.442 times greater risk of HCC than those carrying the A allele $(\mathrm{P}<0.001, \mathrm{OR}=1.442,95 \% \mathrm{CI}: 1.772-1.774)$, indicating that the $\mathrm{C}$ allele mutation was associated with an increased risk of HCC. The detailed results are summarized in Table 2.

We further investigated whether there was a difference in the distribution of the rs2240688 genotype between the clinical subgroups (Table 3). Results showed that the genotype distribution of CD133 rs2240688 was significantly associated with metastasis $(\mathrm{P}=0.008)$. However, the results also showed that the genotype distribution of CD133 rs2240688 was not significantly associated with factors such as age, gender, alcohol consumption, and smoking status $(\mathrm{P}>0.05)$.

We also analyzed common pathological markers of HCC that are routinely tested for, including alpha-fetoprotein (AFP), alanine aminotransferase (ALT), aspartate aminotransferase (AST) and $\gamma$-glutamyl transpeptidase (GGT). Results showed that the genotype distribution of CD133 rs2240688 was significantly associated with ALT $(\mathrm{P}=0.023)$.

\section{Discussion}

Recent research has suggested that CSCs contribute to tumor initiation, metastasis, relapse, and resistance to chemotherapy or radiotherapy (20). SNPs represent the largest proportion of genetic variation in the human genome, and their contribution to cancer susceptibility has been extensively explored $(21,22)$. The CD133-encoding gene is located on human chromosome $4 \mathrm{p} 15$, a region 
Table 1 General characteristics of HCC patients and the normal controls

\begin{tabular}{|c|c|c|c|c|}
\hline Characteristics & Cases $(n=565)$ & Controls $(n=561)$ & $\chi^{2}$ & $P$ value \\
\hline \multicolumn{5}{|l|}{ Age (year) } \\
\hline Range & $10-89$ & $22-78$ & & \\
\hline Mean & 53.62 & 52.15 & & \\
\hline$<40$ & 95 & 102 & 0.365 & 0.546 \\
\hline \multicolumn{5}{|l|}{ Gender } \\
\hline Male & 487 & 488 & 0.562 & 0.453 \\
\hline Female & 78 & 73 & & \\
\hline \multicolumn{5}{|l|}{$\mathrm{BMI}\left(\mathrm{kg} / \mathrm{m}^{2}\right)$} \\
\hline$\geq 24$ & 137 & 151 & & \\
\hline \multicolumn{5}{|l|}{ BCLC stage } \\
\hline$A+B$ stage & 259 & & & \\
\hline$C+D$ stage & 306 & & & \\
\hline \multicolumn{5}{|l|}{ Metastasis } \\
\hline No & 470 & & & \\
\hline Yes & 95 & & & \\
\hline \multicolumn{5}{|l|}{ Smoking status } \\
\hline Yes & 191 & & & \\
\hline \multicolumn{5}{|c|}{ Family history of cancer } \\
\hline No & 487 & & & \\
\hline Yes & 78 & & & \\
\hline \multicolumn{5}{|l|}{ Liver cirrhosis } \\
\hline Absent & 172 & & & \\
\hline Present & 393 & & & \\
\hline \multicolumn{5}{|l|}{ HBV infection } \\
\hline $\mathrm{HbsAg}(-)$ & 57 & & & \\
\hline $\mathrm{HbsAg}(+)$ & 497 & & & \\
\hline HCV infection & 11 & & & \\
\hline
\end{tabular}

HCC, hepatocellular carcinoma; BMI, body mass index; BCLC, Barcelona clinic liver cancer; HBV, hepatitis B virus; HCV, hepatitis C virus. 
Table 2 Comparison of genotype and allele distributions of CD133 rs2240688 in HCC group and controls group

\begin{tabular}{|c|c|c|c|c|c|c|}
\hline Parameter & Case, n (\%) & Controls, n (\%) & OR $(95 \% \mathrm{Cl})$ & $\mathrm{P}_{\mathrm{OR}}$ & $\mathrm{OR}_{\text {adj }}(95 \% \mathrm{Cl})$ & $\mathrm{P}_{\mathrm{adj}}$ \\
\hline \multicolumn{7}{|l|}{ All } \\
\hline$A A$ & $333(58.9)$ & $384(68.4)$ & 1.00 & & 1.00 & \\
\hline $\mathrm{CA}$ & $202(35.7)$ & $159(28.3)$ & $1.465(1.136-1.889)$ & 0.004 & $1.463(1.134-1.887)$ & 0.003 \\
\hline $\mathrm{CA}+\mathrm{CC}$ & $232(41.0)$ & $177(31.5)$ & $1.511(1.180-1.924)$ & 0.001 & $1.508(1.181-1.926)$ & 0.001 \\
\hline \multicolumn{7}{|l|}{ Alleles } \\
\hline$A$ & $868(76.8)$ & $927(82.7)$ & 1.00 & & 1.00 & \\
\hline $\mathrm{C}$ & $262(23.2)$ & $195(17.3)$ & $1.443(1.173-1.775)$ & 0.001 & $1.442(1.772-1.774)$ & 0.001 \\
\hline
\end{tabular}

HCC, hepatocellular carcinoma; OR, odds ratio; $\mathrm{Cl}$, confidence interval.

considered closely related to cancer susceptibility $(18,23,24)$. CD133 is considered an important marker molecule for tumor cells $(17,25,26)$, and O'Brien et al. demonstrated that $\mathrm{CD} 133^{+}$tumor cells have stem cell characteristics (27). A growing number of studies have also demonstrated that CD133 is highly expressed in CSCs of pancreatic ductal adenocarcinoma (PDAC), glioma, colon cancer, gastric cancer, malignant melanoma, non-small cell lung cancer, and other tumors, which suggests that CD133 may play a multifaceted role in tumor development $(9,28-32)$. The prognostic and clinicopathological value of CD133 protein and mRNA expression have also been demonstrated in other studies (33-35). For example, in HCC, subjects with greater CD133 mRNA levels also showed greater invasiveness than subjects with lower CD133 mRNA levels (36). Although it is widely believed that CD133 plays an important role in cancer, the relationship between CD133 polymorphisms and the clinical features of HCC are noticeably lacking. Therefore, in this case control study, we investigated the association of the CD133 SNP rs2240688 with the patient demographics, clinical features, and susceptibility to HCC.

We found that the variant genotypes (AC/CC) of rs2240688 A>C in the miRNA binding site of the stem cell marker gene $C D 133$ were associated with a higher susceptibility to HCC. The distribution frequency of rs2240688 alleles and genotypes in the HCC case group and control group was statistically significant, which is consistent with the results of Liu et al. (37) in lung cancer and Wang et al. (38) in gastric cancer. We found that the CA heterozygous and CC homozygous genotypes, along with $\mathrm{C}$ carrier status and $\mathrm{C}$ alleles, were associated with an increased risk of HCC. This may be attributed to the fact that CD133 expression is closely related to cell proliferation, apoptosis, invasion and metastasis, and angiogenesis (39-42). Furthermore, it has been shown that SNPs located in the 3'untranslated region (3'-UTR) region of the $C D 133$ gene are associated with a variety of human tumors $(38,43,44)$. SNPs in the 3 '-UTR have also been shown to have functional effects on the control of mRNA stability and efficiency through the regulation of miRNA, including miR-34a, -101, -128, -137 and -1385 (45-47). It has been shown that SNPs in a target-binding site can alter the miRNA-mRNA interaction and thus affect the expression of miRNA targets $(48,49)$. Additionally, studies have confirmed that rs2240688 A-to-C transition gains a new binding site of the microRNA has-miR-135a/b, which may play a pivotal role in modulating the effect of the SNP on CD133 expression (38). Interestingly, rs2240688 is located at the 3'-UTR region of the CD133 gene. SNP rs2240688 has been associated with an increased risk of HCC, consistent with the corresponding role of CD133 in promoting the development of liver cancer through other signaling pathways such as $\mathrm{G}$ protein-coupled receptor 87 and CXCL3 (50,51). Additionally, in the subgroup analysis of CD133 rs2240688 and clinical characteristics, our results showed that the genotype distribution of CD133 rs2240688 was significantly associated with metastasis and ALT. Considering the promotional capability of CSCs on tumor growth and metastasis, the present study suggests that CD133 might modify the metastasis competence of HCC via miRNA binding site polymorphisms, which could be a putative target for improved HCC treatment. 
Table 3 Association of CD133 rs2240688 genotype with clinical characteristics in HCC patients

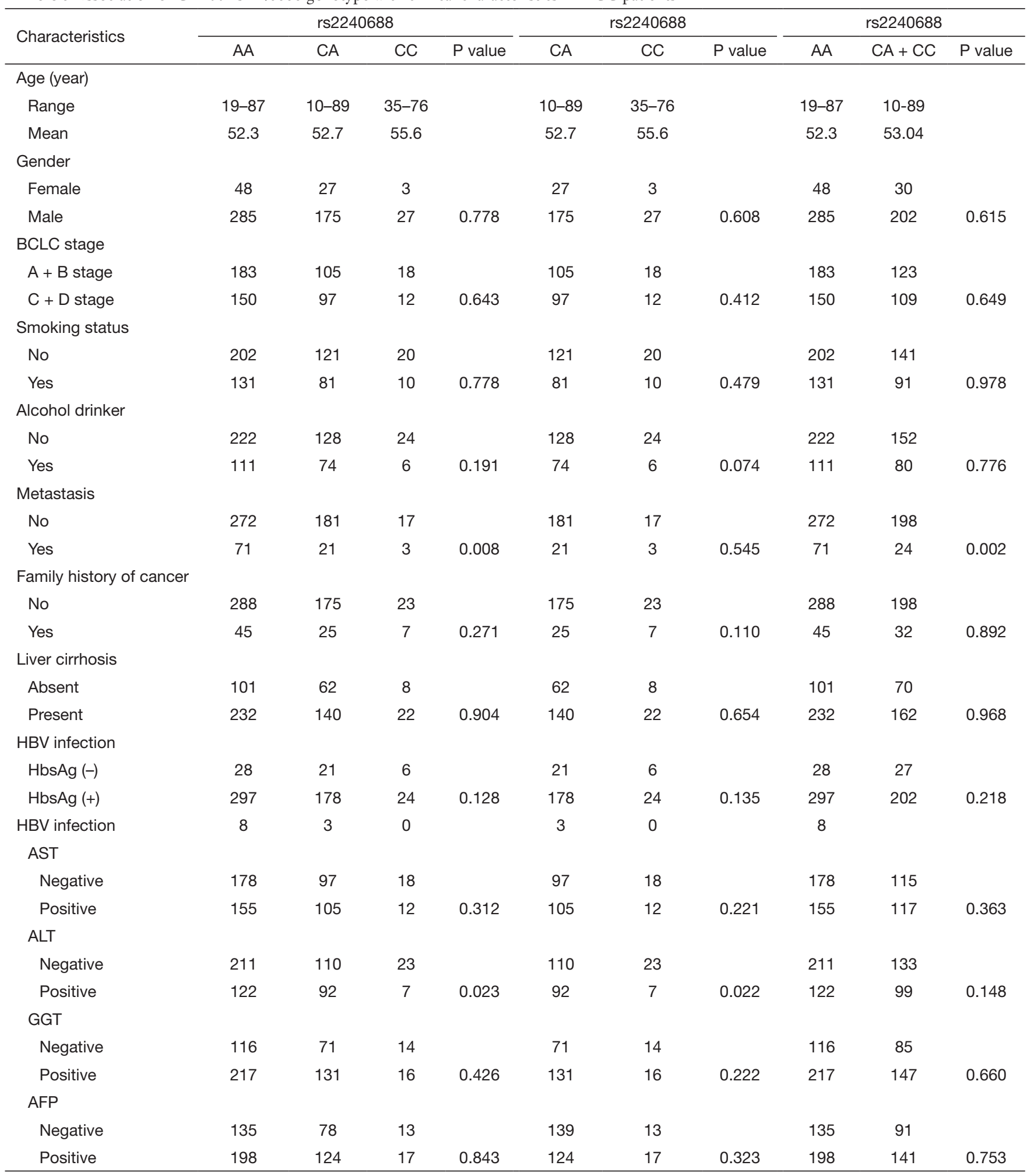

HCC, hepatocellular carcinoma; BCLC, Barcelona clinic liver cancer; HBV, hepatitis B virus; ALT, alanine aminotransferase; AST, aspartate aminotransferase; GGT, $\gamma$-glutamyl transpeptidase; AFP, alpha-fetoprotein. 
In summary, this study was the first to explore the relationship between rs2240688 and the risk of HCC. We found that rs2240688 was associated with an increased risk of HCC and may play an important role in tumor progression, thus providing a basis for the search for novel therapeutic targets. Due to the small sample size of this study, and the inability to obtain more accurate data from the control group, the applicability of these results may be limited. Therefore, future studies investigating more CD133 SNPs, with larger sample sizes and more clinical information, are needed to determine the relationship between CD133 polymorphisms and the risk of developing HCC.

\section{Acknowledgments}

Funding: This work was supported by grants from the National Science Foundation of China (81760530) and the National Science Foundation of Guangxi (2017GXNSFBA198047).

\section{Footnote}

Data Sharing Statement: Available at http://dx.doi. org/10.21037/tcr-19-2690

Conflicts of Interest: All authors have completed the ICMJE uniform disclosure form (available at http://dx.doi. org/10.21037/tcr-19-2690). The authors have no conflicts of interest to declare.

Ethical Statement: The authors are accountable for all aspects of the work in ensuring that questions related to the accuracy or integrity of any part of the work are appropriately investigated and resolved. The study was conducted in accordance with the Declaration of Helsinki (as revised in 2013). This study was approved by the ethics committee of the Affiliated Tumor Hospital of Guangxi Medical University (approval ID: LW2020007). Written informed consent was provided by all subjects in the study.

Open Access Statement: This is an Open Access article distributed in accordance with the Creative Commons Attribution-NonCommercial-NoDerivs 4.0 International License (CC BY-NC-ND 4.0), which permits the noncommercial replication and distribution of the article with the strict proviso that no changes or edits are made and the original work is properly cited (including links to both the formal publication through the relevant DOI and the license). See: https://creativecommons.org/licenses/by-nc-nd/4.0/.

\section{References}

1. Weng CJ, Hsieh YH, Tsai CM, et al. Relationship of insulin-like growth factors system gene polymorphisms with the susceptibility and pathological development of hepatocellular carcinoma. Ann Surg Oncol 2010;17:1808-15.

2. Yang Y, Huang H, Wang W, et al. Association of insulin growth factor-1 receptor gene polymorphisms with genetic susceptibility to idiopathic short stature. Genet Mol Res 2013;12:4768-79.

3. Canavese $\mathrm{M}, \mathrm{W}$ ijesundara $\mathrm{D}$, Maddern GJ, et al. Hepatitis $C$ virus drives the pathogenesis of hepatocellular carcinoma: from immune evasion to carcinogenesis. Clin Transl Immunology 2016;5:e101.

4. Kim J, Choi SJ, Lee SH, et al. Predicting survival using pretreatment CT for patients with hepatocellular carcinoma treated with transarterial chemoembolization: comparison of models using radiomics. AJR Am J Roentgenol 2018;211:1026-34.

5. Batlle E, Clevers H. Cancer stem cells revisited. Nat Med 2017;23:1124-34.

6. Wang R, Chen S, Li C, et al. Fusion with stem cell makes the hepatocellular carcinoma cells similar to liver tumorinitiating cells. BMC Cancer 2016;16:56.

7. Wang $\mathrm{Y}, \mathrm{Wu} \mathrm{G}, \mathrm{Fu} \mathrm{X}$, et al. Aquaporin 3 maintains the stemness of CD133+ hepatocellular carcinoma cells by activating STAT3. Cell Death Dis 2019;10:465.

8. Corbeil D. Prominin-1 (CD133): new insights on stem \& cancer stem cell biology. New York: Springer-Verlag, 2013:VII-IX.

9. Huang R, Mo D, Wu J, et al. CD133 expression correlates with clinicopathologic features and poor prognosis of colorectal cancer patients: an updated meta-analysis of 37 studies. Medicine (Baltimore). 2018;97:e10446.

10. Song S, Pei G, Du Y, et al. Interaction between CD133 and PI3K-p85 promotes chemoresistance in gastric cancer cells. Am J Transl Res 2018;10:304-14.

11. Long Q, Yang R, Lu W, et al. Adenovirus-mediated truncated Bid overexpression induced by the Cre/LoxP system promotes the cell apoptosis of CD133+ ovarian cancer stem cells. Oncol Rep 2017;37:155-62.

12. Feng D, Gong P, Li W, et al. Identification and characterization of tumorigenic liver cancer stem cells by CD133 and CD24. J Biomater Tissue Eng 2015;5:635-46. 
13. Guo X, Li D, Chen Y, et al. SNP rs2057482 in HIF1A gene predicts clinical outcome of aggressive hepatocellular carcinoma patients after surgery. Sci Rep 2015;5:11846.

14. Li H, Liu F, Zhu H, et al. Interaction between polymorphisms of IFN- $\gamma$ and MICA correlated with hepatocellular carcinoma. Med Sci Monit 2016;22:549-53.

15. Shaker O, Alhelf M, Morcos G, et al. miRNA-101-1 and miRNA-221 expressions and their polymorphisms as biomarkers for early diagnosis of hepatocellular carcinoma. Infect Genet Evol 2017;51:173-81.

16. Li Z, Zhang Z, He Z, et al. A partition-ligationcombination-subdivision EM algorithm for haplotype inference with multiallelic markers: update of the SHEsis (http://analysis.bio-x.cn). Cell Res 2009;19:519-23.

17. Chen YL, Lin PY, Ming YZ, et al. The effects of the location of cancer stem cell marker CD133 on the prognosis of hepatocellular carcinoma patients. BMC Cancer 2017; 17:474.

18. Hu N, Wang C, Ng D, et al. Genomic characterization of esophageal squamous cell carcinoma from a high-risk population in China. Cancer Res 2009;69:5908-17.

19. Mehta B, Daniel R, Phillips C, et al. Forensically relevant SNaPshot ${ }^{\circledR}$ assays for human DNA SNP analysis: a review. Int J Legal Med 2017;131:21-37.

20. Reya T, Morrison SJ, Clarke MF, et al. Stem cells, cancer, and cancer stem cells. Nature 2001;414:105-11.

21. Easton DF, Pooley KA, Dunning AM, et al. Genomewide association study identifies novel breast cancer susceptibility loci. Nature 2007;447:1087-93.

22. Hong SN, Park C, Kim JI, et al. Colorectal cancersusceptibility single-nucleotide polymorphisms in Korean population. J Gastroenterol Hepatol 2015;30:849-57.

23. Feng BJ, Huang W, Shugart YY, et al. Genome-wide scan for familial nasopharyngeal carcinoma reveals evidence of linkage to chromosome 4. Nat Genet 2002;31:395-9.

24. Petersen S, Aninat-Meyer M, Schlüns K, et al. Chromosomal alterations in the clonal evolution to the metastatic stage of squamous cell carcinomas of the lung. Br J Cancer 2000;82:65-73.

25. Janikova M, Skarda J, Dziechciarkova M, et al. Identification of CD133+/nestin+ putative cancer stem cells in non-small cell lung cancer. Biomed Pap Med Fac Univ Palacky Olomouc Czech Repub 2010;154:321-6.

26. Ren F, Sheng WQ, Du X. CD133: a cancer stem cells marker, is used in colorectal cancers. World J Gastroenterol 2013;19:2603-11.

27. O'Brien CA, Pollett A, Gallinger S, et al. A human colon cancer cell capable of initiating tumour growth in immunodeficient mice. Nature 2007;445:106-10.

28. Madjd Z, Erfani E, Gheytanchi E, et al. Expression of CD133 cancer stem cell marker in melanoma: a systematic review and meta-analysis. Int J Biol Markers 2016;31:e118-25.

29. Li X, Zhao H, Gu J, et al. Prognostic value of cancer stem cell marker CD133 expression in pancreatic ductal adenocarcinoma (PDAC): a systematic review and metaanalysis. Int J Clin Exp Pathol 2015;8:12084-92.

30. Wu B, Sun C, Feng F, et al. Do relevant markers of cancer stem cells CD133 and Nestin indicate a poor prognosis in glioma patients? A systematic review and meta-analysis. J Exp Clin Cancer Res 2015;34:44.

31. Wen L, Chen XZ, Yang K, et al. Prognostic value of cancer stem cell marker CD133 expression in gastric cancer: a systematic review. PLoS One 2013;8:e59154.

32. Wu H, Qi XW, Yan GN, et al. Is CD133 expression a prognostic biomarker of non-small-cell lung cancer? A systematic review and meta-analysis. PLoS One. 2014;9:e100168.

33. Huh JW, Park YS, Lee JH, et al. CD133 mRNA expression and microsatellite instability in colorectal carcinoma. J Surg Oncol 2010;102:765-70.

34. Lee MR, Ji SY, Mia-Jan K, et al. Chemoresistance of CD133(+) colon cancer may be related with increased survivin expression. Biochem Biophys Res Commun 2015;463:229-34.

35. Fathi A, Mosaad H, Hussein S, et al. Prognostic significance of CD133 and ezrin expression in colorectal carcinoma. IUBMB Life 2017;69:328-40.

36. Jia Q, Zhang X, Deng T, et al. Positive correlation of Oct4 and ABCG2 to chemotherapeutic resistance in CD90(+)CD133(+) liver cancer stem cells. Cell Reprogram 2013;15:143-50.

37. Liu QF, Zhang ZF, Hou GJ, et al. Polymorphisms of the stem cell marker gene CD133 and the risk of lung cancer in Chinese population. Lung 2016;194:393-400.

38. Wang Q, Liu H, Xiong H, et al. Polymorphisms at the microRNA binding-site of the stem cell marker gene CD133 modify susceptibility to and survival of gastric cancer. Mol Carcinog 2015;54:449-58.

39. Jang JW, Song Y, Kim SH, et al. CD133 confers cancer stem-like cell properties by stabilizing EGFR-AKT signaling in hepatocellular carcinoma. Cancer Lett 2017;389:1-10.

40. Liu C, Li Y, Xing Y, et al. The interaction between cancer stem cell marker CD133 and src protein promotes focal adhesion kinase (FAK) phosphorylation and cell migration. 
J Biol Chem 2016;291:15540-50.

41. Wei Y, Jiang Y, Zou F, et al. Activation of PI3K/Akt pathway by CD133-p85 interaction promotes tumorigenic capacity of glioma stem cells. Proc Natl Acad Sci U S A 2013;110:6829-34.

42. Park EK, Lee JC, Park JW, et al. Transcriptional repression of cancer stem cell marker CD133 by tumor suppressor p53. Cell Death Dis 2015;6:e1964.

43. Liu QF, Zhang ZF, Hou GJ, et al. Polymorphisms of the stem cell marker gene CD133 are associated the clinical outcome in a cohort of Chinese non-small cell lung cancer patients. BMJ Open 2017;7:e016913.

44. Aravantinos G, Isaakidou A, Karantanos T, et al. Association of CD133 polymorphisms and response to bevacizumab in patients with metastatic colorectal cancer. Cancer Biomark 2015;15:843-50.

45. Ma T, Liu X, Cen Z, et al. MicroRNA-302b negatively regulates IL-1beta production in response to MSU crystals by targeting IRAK4 and EphA2. Arthritis Res Ther 2018;20:34.

46. Zhang L, Yang CS, Varelas X, et al. Altered RNA editing in 3' UTR perturbs microRNA-mediated regulation of

Cite this article as: Pan X, Huang L, Mo D, Liang Y, Huang Z, Zhu B, Fang M. SNP rs2240688 in CD133 gene on susceptibility and clinicopathological features of hepatocellular carcinoma. Transl Cancer Res 2020;9(10):5940-5948. doi: $10.21037 /$ tcr-19-2690 oncogenes and tumor-suppressors. Sci Rep 2016;6:23226.

47. Vishnoi A, Rani S. MiRNA biogenesis and regulation of diseases: an overview. Methods Mol Biol 2017;1509:1-10.

48. Vallender EJ, Priddy CM, Hakim S, et al. Functional variation in the $3^{\prime}$ untranslated region of the serotonin transporter in human and rhesus macaque. Genes Brain Behav 2008;7:690-7.

49. Yao $\mathrm{Y}$, Shao J, Wu J, et al. The functional variant in the 3'UTR of PTPRT with the risk of esophageal squamous cell carcinoma in a Chinese population. Cell Physiol Biochem 2015;36:306-14.

50. Yan M, Li H, Zhu M, et al. G protein-coupled receptor 87 (GPR87) promotes the growth and metastasis of CD133(+) cancer stem-like cells in hepatocellular carcinoma. PLoS One 2013;8:e61056.

51. Zhang L, Zhang L, Li H, et al. CXCL3 contributes to CD133(+) CSCs maintenance and forms a positive feedback regulation loop with CD133 in HCC via Erk1/2 phosphorylation. Sci Rep 2016;6:27426.

(English Language Editors: C. Betlazar-Maseh and J. Gray) 